\title{
Pedagogías constructoras de paces en clave decolonial. La experiencia de la Universidad Veracruzana Intercultural
}

\section{Peace constructing pedagogies in decolonial terms. The experience of the Universidad Veracruzana Intercultural}

\author{
VERónica Moreno URIBE* \\ GUNTHER DIETZ $^{* *}$
}

A la luz de las claves analíticas que los estudios interculturales y el feminismo decolonial proponen, analizamos la experiencia de la Universidad Veracruzana Intercultural (UVI), sede Grandes Montañas, en la construcción de una pedagogía detonante de relaciones empáticas y restauradora de vínculos comunitarios que derivan en formas de relacionamiento que gestan bienestar y permiten la resolución de conflictos. Desde una perspectiva que apela al reconocimiento de los códigos de significación locales y territorializados sobre paces/violencias, exploramos, con un enfoque interactoral, bilingüe e intercultural, las nociones y representaciones que niños, niñas y jóvenes nahuas y mestizos, vinculados a la UVI, tienen en torno a ellas; esto, a partir de cuatro horizontes de sentido y acción: el interpersonal, el comunitario, el escolar y el familiar. En ello consideramos las prácticas para gestionar bienestar y dirimir conflicto, interpeladas por la apuesta pedagógico-política de la UVI en la región de las Grandes Montañas en Veracruz, México.

In light of the analytical keys that intercultural studies and decolonial feminism provide, we analyze the experience of the Universidad Veracruzana Intercultural (UVI), campus Grandes Montañas, in the construction of a pedagogy that triggers empathic relations and restores community bonds that promote forms of relationship which generate well-being and contribute to conflict resolution. From a perspective that appeals to the recognition of local and territorialized codes of meaning regarding peace/violence, the notions and representations that Nahua and mestizo children and young people related to the UVI have, are explored from a bilingual and intercultural approach which considers the diversity of actors involved through four horizons of meaning and action: An interpersonal one, a community one, a school horizon and a family horizon regarding in each of them practices to manage well-being and to resolve conflicts, which are challenged by the political pedagogical commitment of the UVI in the Grandes Montañas region of Veracruz, Mexico.

\section{Palabras clave:}

paces/violencias, interculturalidad, feminismo

decolonial
Keywords:
peace/violence, interculturality, decolonial
feminism

Recibido: 31 de agosto de 2018.| Aceptado para su publicación: 25 de enero de 2018. Recuperado de: https://sinectica.iteso.mx/index.php/SINECTICA/article/view/902 DOI: 10.31391/S2007-7033(2019)0052-005

\footnotetext{
* Doctora en Sociología por la Benemérita Universidad Autónoma de Puebla. Coordinadora de Investigación en la Universidad Veracruzana Intercultural. Líneas de investigación: salud sexual y reproductiva, pobreza, desarrollo y derechos y economía, temas que ha trabajado desde la teoría de género y feminista. Correo electrónico: veronic7@hotmail.com / http://orcid.org/0000-0002-6549-6874

${ }^{* *}$ Doctor en Antropología por la Universidad de Hamburgo (Alemania). Investigador titular en el Instituto de Investigaciones en Educación de la Universidad Veracruzana (Xalapa, México). Líneas de investigación: estudios interculturales y educación intercultural. Correo electrónico: guntherdietz@gmail.com / http://orcid.org/0000-0002-1487-2673
} 


\section{INTRODUCCIÓN}

$7 \mathrm{n}$ el estado de Veracruz, México, en 2018, murieron mensualmente en for-

$\mathrm{E}$ ma violenta un aproximado de 19 mujeres, por homicidios o feminicidios (Universidad Veracruzana, s.f.). De acuerdo con datos del Secretariado Ejecutivo del Sistema Nacional de Seguridad Pública, la entidad ocupa el primer lugar a nivel nacional en casos de feminicidio y concentra, con otras cinco, el $40 \%$ de los decesos por razones de género en el territorio (Delgado, 2018). Desde una diversidad de enfoques y disciplinas, se ha generado un acervo considerable de diagnósticos que describen la multiplicidad de dispositivos mediante los cuales se crea y reproduce violencia y el impacto de ello en la vida de las personas, las comunidades y las instituciones.

Pocos son, sin embargo, los esfuerzos por explorar, desde una mirada intercultural y decolonial, las nociones particulares y territorializadas sobre la violencia que tienen diferentes grupos sociales, así como las respuestas construidas para gestionarla y detonar bienestar.

Este texto es resultado del proyecto de investigación "Educación para la paz. Descolonialidad, interculturalidad y género apuestas teórico políticas para la intervención en contextos de violencias. Una propuesta de investigación vinculada", desarrollado como parte de una estancia posdoctoral en el Instituto de Investigaciones en Educación de la Universidad Veracruzana.

En el ámbito de la educación, es reciente la proliferación de estudios que dilucidan mecanismos para la construcción de formas de relacionamiento conducentes a dirimir conflictos y tendentes a fortalecer prácticas y actitudes consideradas detonadoras de relaciones pacíficas. Así, la educación para la paz es un campo de estudio emergente, el cual -sobre todo desde Abya Yala (nombre dado por el pueblo kuna a América, cfr. Gargallo, 2015)- ha estado anclado a las indagaciones derivadas del posconflicto, que buscan restaurar los vínculos y las relaciones rasgadas por la violencia estructural, directa y cultural (Galtung, 2003).

Si bien no es objeto de este artículo discutir en detalle la perspectiva de educación para la paz de la que abrevó el proyecto de investigación que le dio origen, vale la pena mencionar que son dos fuentes las que se conjugaron para proponer un acercamiento a los estudios de paces y violencias; por una parte, la perspectiva de no violencia (ahimsa en sánscrito) y de interdependencia desarrollada por Mahatma Gandhi en el Programa Constructivo (Gandhi, 2016; para conocer el contexto histórico de los postulados, cfr. UNAM, s.f.) y por otra, la perspectiva de paz territorializada, de Alejandro Pimienta y Raquel Pulgarín (2017).

A pesar de la necesidad de identificar la constelación de elementos que se conjugan para configurar conflictividad en los contextos concretos, encontramos, sin embargo, que la presencia de propuestas desde la pedagogía, ocupadas en construir coordenadas de una "educación para la paz" situada y territorializada, es aún exigua. Esta falencia dificulta derivar formulaciones que partan de esta especificidad situada y construir estrategias de resolución desde los marcos de sentido y significación propios de las comunidades donde el conflicto acaece. Más escasos son los estudios que indaguen sobre los códigos y las nociones locales de conflicto y paces, y pongan con ello a discusión los discursos hegemónicos sobre la violencia y la paz. 
En este texto, resumimos, de manera parcial, parte de los hallazgos del proyecto de investigación realizado en la sede Grandes Montañas de la Universidad Veracruzana Intercultural (UVI), con estudiantes provenientes de pueblos nahuas y mestizos de la región de Zongolica, Veracruz, y sus grupos de vinculación.

La UVI se crea en 2005 como entidad académica de la Universidad Veracruzana y es la única de las once universidades interculturales mexicanas emergidas en el seno de una institución de educación superior. Se asienta en cinco regiones del estado de Veracruz: Huasteca, Selvas, Totonacapan, Grandes Montañas, donde se desarrollan dos programas educativos (licenciatura en Gestión Intercultural para el Desarrollo y licenciatura en Derecho Intercultural con enfoque de Pluralismo Jurídico), así como una oficina de gestoría académica y administrativa en la ciudad de Xalapa. Actualmente, atiende una matrícula de 349 estudiantes, hablantes de diez lenguas originarias diferentes al español.

La investigación indaga las nociones y representaciones que estudiantes de la UVI y sus grupos de vinculación confieren a la paz y a la violencia, a partir de cuatro horizontes de sentido y acción: el interpersonal, el comunitario, el escolar y el familiar. Propone una discusión a la luz de los postulados de la decolonialidad y del feminismo que de él abreva, que interpela las teorías hegemónicas sobre educación para la paz y analiza la impronta que la educación con enfoque intercultural tiene en la articulación de una pedagogía para la empatía, potencialmente detonadora de bienestar y para la resolución de conflictos.

Este artículo parte de la pregunta articuladora sobre los aportes del enfoque intercultural en educación a la elucidación de las formas locales para gestionar y dirimir conflictos y comprender la paz; problematiza las formas en las que en el sistema del mundo moderno, capitalista, colonial y patriarcal se interconectan sistemas diferenciados de dominación, que suman y engranan violencias y se expresan en relaciones de opresión, explotación, despojo, discriminación y otras, cuya complejidad requiere ser abordada de manera interseccional (Crenshaw 2012; Lugones 2012).

Desde estas claves, nos situamos en forma convergente como académicos que han tenido la oportunidad de participar en el proceso de diseño del proyecto pedagógico intercultural desde sus inicios, amén de las trayectorias específicas de cada quien, en la antropología feminista y de la educación. Esto nos permite identificar cómo se instalan las lógicas coloniales y patriarcales en la construcción de conocimiento y su validación, y posicionarnos al respecto, distanciándonos de ellas y asumiendo una perspectiva de la investigación comprometida socialmente, que derive en la posibilidad de mejorar las vidas de las personas con las que se gesta.

En términos metodológicos, conjuga las premisas de la investigación vinculada para la gestión desarrolladas por la UVI (Universidad Veracruzana-UV-Intercultural, s.f.), las cuales propenden por el pluralismo epistémico, que ponga en diálogo lógicas diferenciadas de comprender, nombrar y representar el mundo, así como la interlocución entre actores comunitarios y académicos en el proceso de investigación, la incorporación de los enfoques intercultural, interlingüe y de género, para la comprensión compleja y situada de la realidad, y la pertinencia y relevancia de la investigación para el mejoramiento de las condiciones de vida de los habitantes 
concernidos en ella. Estas premisas se sustentan en la consideración de que la investigación con poblaciones históricamente excluidas contiene una responsabilidad ética y política y un compromiso social.

Trabajamos por medio de talleres con siete grupos de estudiantes de diferentes semestres de la licenciatura en Gestión Intercultural para el Desarrollo de la UVI y tres de sus grupos de vinculación, pertenecientes a escuelas primarias de los municipios de Tequila y Tlaquilpa, en la Sierra de Zongolica. Con estos últimos, desarrollamos juegos escénicos con títeres, liderados por egresadas y estudiantes bilingües de la UVI, y entrevistas a partir de dibujos elaborados por las niñas y los niños.

\section{PACES Y VIOLENCIAS EN CLAVE DECOLONIAL E INTERCULTURAL}

Recuperamos tres claves analíticas de los estudios decoloniales e interculturales, y en particular del feminismo decolonial, para problematizar cómo se configuran las violencias en el sistema mundo moderno, capitalista, colonial y patriarcal, para derivar de ello una reflexión en torno a las condiciones de posibilidad de pedagogías pacifistas. Retomamos de manera preponderante los postulados desarrollados por Rita Segato $(2006,2007,2011,2016)$ y, en menor medida, por María Lugones (2012), Sylvia Marcos (2014) y otras autoras latinoamericanas, relativos a la necesidad de interconectar las categorías de género y raza para comprender cómo se configuran y expresan las relaciones de dominación y que abrevan sustantivamente de los feminismos de color (Jabardo, 2014), de autoras como bell hooks (2004), Patricia Hill Collins, Ángela Davis y otras, que aportaron a la perspectiva interseccional.

La primera clave analítica, de orden epistemológico y ontológico, plantea que la producción de conocimiento a partir de las coordenadas del patrón de poder moderno/capitalista está al servicio del mercado, se valúa en términos del sistema de valor dinero y es profundamente excluyente de racionalidades "no occidentales" (Grosfoguel, 2013). Como lo expresa Espinosa (2014),

... la opresión se fundamenta en un sistema de conocimiento y producción del mundo de la vida, un sistema de clasificación social, dentro del cual han surgido las categorías dominantes de opresión (género, raza, clase), sistema instituido a través de la empresa colonizadora y la razón imperial a su servicio, ha llegado el momento de una desobediencia epistémica amplia que derrumbe el armazón de compresión del mundo tal cual lo ha producido y ha sido impuesto por la modernidad occidental (p. 7).

Es de cuño colonial, en tanto que instala códigos de relacionamiento y sentido a partir de políticas raciales, que, a su vez, configuran estructuras de clase (hooks, 2004), diferenciadas por género; con ello determina quiénes son los sujetos certificados para producir conocimiento válido. Bajo su tutela, se instituye, de manera unívoca, una racionalidad binaria, maniquea y excluyente de organización y representación de la realidad, que genera profundas escisiones y rivalidades entre las personas al jerarquizarlas y que, a decir de Segato (2011) y Gutiérrez (2014), fragmenta el mundo en dos, al instaurar una lógica de significación del "neutro ordenador universal" a partir de la cual lo "otro" relacional deviene resto:

... a partir de la modernidad europea ligada a la expansión del capital, se establece paulatinamente lo masculino como central y única medida de lo humano. Los siglos XIV, XV y XVI no son las primeras épocas históricas en que esto ocurre; podemos rastrear tanto los usos como los significados materiales, simbólicos y formales de instituir al "varón" 
como representante de "lo humano", al menos desde la Grecia clásica. Sin embargo, la operación plenamente moderna consiste en asimilar lo masculino al capital y éste a lo "neutro". Un supuesto "neutro" ordenador -claramente masculino en tanto es no femenino- que instala un eje de enunciación universal afirmativo: "El Hombre". El arreglo lógico, semántico que se produce una vez instituido este "neutro ordenador" universal afirmativo -masculino en tanto claramente no femenino- consiste en el arrinconamiento de todo lo demás en un ambiguo y opaco lugar de lo particular -o más bien de múltiples particulares ahora carentes de medida propia-... (Gutiérrez, 2014, pp. 89-90).

Esos otros, restos racializados en tanto no blancos, feminizados, en tanto no varones, improductivos, en tanto no proveedores, incivilizados, en tanto no eurocentrados, son sujetos prescindibles, mermados en su densidad ontológica por cuanto deben ser mediados para alcanzar existencia; disminuidos en su capacidad para enunciar el mundo y desautorizados para gestar información sobre él a partir de su propia experiencia. Interdictos en su condición de resto, aquellas y aquellos otrificados, y "carentes de medida propia", se encuentran incompletos para denominar el mundo con un vocabulario propio y desde su experiencia.

En este sentido, la colonialidad del poder es de orden patriarcal, dado que la mediación que impone a los sujetos exiliados del uno ordenador desprovee de valor y densidad ontológica a aquellos sujetos "restos", femeninos o feminizados, que en ese procedimiento son minorizados (Segato, 2016); a razón de ello, son desautorizados para nombrar el mundo y producir conocimiento sobre él. Así, no solo le resta densidad a los sujetos (Moreno, 2016), al despolitizarlos, sino también a sus conocimientos, experiencias y prácticas; a contrapelo, aquellos legitimados para generar conocimiento son quienes discursan en la gramática propia de las disciplinas, hablan lenguas hegemónicas y son por antonomasia varones heterosexuales blancos y productivos que circulan en la esfera de lo público y no tienen que disputarse, más que entre ellos mismos, un lugar en el conclave del conocimiento legítimo.

Así, la sinergia patriarcado-capitalismo ha sido ampliamente trabajada desde la economía feminista (Pérez, 2014; Federici, 2010), el feminismo comunitario (Gutiérrez, 2014), el feminismo decolonial (Segato, 2011, 2016; Espinosa, 2014; Espinosa et al., 2014), la teoría crítica del patriarcado (Von Werlhof y Behmann, 2010) y del desarrollo (Mohanty, 2008) y ha permitido comprender -entre otras razones- cómo se articulan para configurar un sistema complejo que despoja de valor y densidad a todo aquello que feminiza: saberes, haceres, experiencias, trabajos, mediante la operación expuesta, consistente en expurgar, desautorizar y deslegitimar a lo que construye como "resto".

A pesar de la heterogénea performatividad del género, es decir, de su maleable transformación histórica y su variable expresión y representación en el tiempo y espacio, se constituyen patrones continuos, que, tal como explica Assis (2018), están mediados por políticas de masculinización, sobre todo para poblaciones racializadas, mediante estrategias y dispositivos tendentes a construir un patrón de género colonial y capitalista masculinizado, que, aunque no lo determine de modo irrestricto en todos los contextos, es condicionante en "grados variables".

La segunda clave la proporciona el concepto de interseccionalidad (Crenshaw, 2012; Lugones, 2008, 2012), utilizado originariamente por teóricas de los feminismos negros para incorporar el tema de la opresión de género, otras formas de dominación como el racismo y las relaciones de clase: 
Lideradas por las afroamericanas, todas las mujeres ni eurocentradas ni blancas estamos luchando para que la interseccionalidad se vuelva una característica metodológica necesaria de los estudios de género: raza, clase y género son inseparables y la intersección de las categorías homogéneas dominantes que borran la heterogeneidad interna y borran a la afroamericana, la afrocaribeña, la cherokee, la siux, la navajo, la africana, la indocaribeña, la afrocolombiana, la afrolatinoamericana, la guaraní, la mapuche, la aymara, la toba, la quechua (Lugones, 2012, p. 134).

Retomado más adelante por los feminismos decoloniales, este concepto nos ayuda a comprender cómo en el patrón de poder colonial se imbrican sistemas diferenciados de dominación que, en su interconexión, agudizan las formas en las que el poder se simboliza, materializa y expresa. Analizar desde esta propuesta una relación de explotación, por ejemplo, requiere identificar el lugar social de las sujetas y los sujetos específicos y la concepción que del trabajo se tiene en el contexto según quien realice, para mirar cómo en el capitalismo existen formas diferenciadas de explotación, a razón de las políticas raciales imperantes, del pasaporte que porte, de la lengua que hable, o de la edad que tenga. Deja de ser en abstracto la explotación, siguiendo nuestro ejemplo, un fenómeno que describe la relación capital-trabajo para concretarse, indagado desde la interseccionalidad, en una compleja constelación de formas en las que el poder se interconecta y se encarna en sujetas y sujetos concretos.

La tercera clave analítica la proporciona la noción de interculturalidad desarrollada por los estudios interculturales (Dietz, 2017). Superando nociones esencializantes y culturalistas de la diversidad y reconociendo la interseccionalidad de las identidades que asumen los sujetos inmersos en relaciones históricamente desiguales y asimétricas (Gundara, 2000), la interculturalidad que aquí proponemos analiza cómo interactúan estas y estos sujetos y qué pautas de articulación, apropiación y resistencia despliegan hacia fuera de sus "culturas íntimas" (Lomnitz, 1995), de los espacios culturales o comunitarios construidos y defendidos desde inicios de la colonización frente a actores exógenos y hegemónicos.

Desde esta noción de interculturalidad, muy alejada del hegemónico "multiculturalismo neoliberal" (Hale, 2002), analizamos la naturaleza conflictiva y colonial/moderna que revisten las relaciones interculturales entre colectividades y sujeto/as, así como las potencialidades de establecer "diálogos de saberes" por encima de las desigualdades y asimetrías entre, por ejemplo, tradiciones y prácticas diversas de pluralismo jurídico y de "interlegalidad" (Sierra, 2004). Una universidad que se autodenomina intercultural aspira a descolonizar estas relaciones jurídicas, culturales, lingüísticas y epistémicas, y abrir espacios de intercambios entre saberes académicos y comunitarios.

¿Qué implica mirar la construcción de paz y los procesos pedagógicos relativos a ella considerando estas claves analíticas? Encontramos tres tendencias problemáticas en los estudios de educación para la paz, que, desde nuestra perspectiva, limitan el alcance pedagógico y político de sus propuestas (para conocer diversos estudios y reflexiones sobre estas tendencias, ver Galtung [2003] y Ameglio y Ramírez [2016]):

- La primera es proclive a proponer estrategias universales, que parten de presupuestos eurocentrados y derivados de problemáticas ajenas, para la resolución de conflictos, independientemente del contexto, el territorio y las relaciones interculturales concretas. 
- La segunda está vinculada a la anterior y tiende a omitir las representaciones y los sentidos que los sujetos/as -genérica, cultural y lingüísticamente- confieren a las paces y las violencias, con base en las cuales gestionan prácticas para dirimir conflictos y gestar bienestar en sus congregaciones.

- La tercera es propensa a disociar en categorías estáticas y cerradas los fenómenos conducentes a la generación de violencia. Esta categorización fragmentada impide comprender la realidad de suyo interconectada.

\section{HACIA UNA INVESTIGACIÓN VINCULADA: PROPUESTA METODOLÓGICA}

La indagación fue realizada entre agosto de 2017 y julio de 2018 en la sede de Grandes Montañas de la UVI; fue parte del trabajo de investigación desarrollado durante la estancia posdoctoral en el Instituto de Investigaciones en Educación de la Universidad Veracruzana.

Organizamos dos tipos de talleres: el primero con grupos de vinculación de estudiantes o egresadas de la UVI, conformados por 37 niñas y niños entre tercero y quinto grado de primaria, y el segundo, con grupos de estudiantes de la licenciatura en Gestión Intercultural para el Desarrollo de la UVI, en total 56. Los grupos de vinculación están compuestos por asociaciones comunitarias de diferente naturaleza (entre ellas, educativas) y son aquellos con los que los alumnos/as de la UVI deciden trabajar su documento recepcional desde el enfoque de la investigación vinculada.

En el primer caso, los talleres se llevaron a cabo en dos escuelas primarias: la Indígena Gabino Barreda, del Barrio de Tolapa, en Tequila, Veracruz, y la Adolfo López Mateos, de la comunidad de Tequila y con niñas de la primaria indígena de Tlaquilpa, en el marco del Festival por el Buen Vivir, organizado por la UVI. En el desarrollo de los ejercicios planteados en estos primeros talleres fue fundamental el acompañamiento de las egresadas de la UVI Verónica Lizeth Andrade Pérez y Angélica Rosas de la Cruz (en el caso de la primaria de Tolapa), dado que ellas tienen un trabajo sistemático con los niños y las niñas a partir de su intervención con el Colectivo de Teatro Comunitario de Tequila, y de la estudiante Gabriela Citlahua, en la primaria de Tequila.

En estos talleres recurrimos a tres técnicas para la identificación de representaciones y prácticas en torno a las paces/violencias: el juego escénico, la elaboración de títeres junto con la construcción de historias y el dibujo entrevista; todas ellas son propuestas metodológicas que le dan agencia política a niños y niñas para interpretar e incidir en su realidad (Podestá, 2007; Núñez, 2017) y mediante las cuales es posible identificar los procesos de negociación de los significados que las personas participantes quieren conferir a las narraciones.

En todos los casos, les pedimos a los grupos que imaginaran una historia con una característica peculiar: en algún momento de la trama los eventos se complicarían y darían lugar a un conflicto o problema que generaría malestar entre los personajes, el cual, posteriormente, se resolvería. Les solicitamos que propusieran personajes que detonaran el conflicto y otros que ayudaran a reparar el daño ocasionado. No les dimos ninguna indicación sobre el tipo de conflicto ni sobre los personajes, el contexto, el ámbito o las formas de resolución. Tampoco les indicamos que las representaciones tendrían que ser en español; parte de ellas, sobre todo las de los juegos 
escénicos, fueron en náhuatl. En este caso, es importante precisar que la conducción del ejercicio estuvo a cargo de las egresadas de la UVI, quienes iban traduciendo al español el intercambio que sostenían con las niñas y los niños.

Los elementos observados en estos primeros talleres fueron:

-Horizontes de sentido y acción: comunitario, familiar, interpersonal, escolar.

-Nociones de paces/violencias, representaciones asociadas a estas.

-Situación que inocula conflicto y situación que lo repara o resuelve.

-Personajes y roles que detonan el conflicto y que lo reparan o resuelven.

-Prácticas, relaciones y símbolos asociados a la paz y el conflicto.

El segundo tipo de talleres fue realizado con los estudiantes de la UVI de segundo, cuarto y sexto semestre, gran parte de ellos bilingües. Estos talleres se centraron en explorar una dimensión de la violencia que, para los jóvenes, se encuentra en la totalidad de los ámbitos de la vida comunitaria y que se presenta de manera constante en los contextos donde ellos tienen interacciones; se trata de la violencia de género, que, en intersección con los procesos de racialización y discriminación por razones étnico-lingüísticas, de clase y de edad, configura un escenario por demás complejo.

Los siete talleres desarrollados con los estudiantes se organizaron a partir de varios ejes de análisis:

-Identificación de las instituciones, los discursos y los dispositivos por medio de los cuales se conducen y reproducen los procesos de construcción de género.

-Identificación de los sistemas que se imbrican para generar violencia y que se expresan en formas diferenciadas de dominación en el horizonte de la modernidad colonial, capitalista y patriarcal, como opresión/despojo/explotación/discriminación.

-Identificación y análisis, desde una perspectiva intercultural, de las nociones de paces y violencias, sus representaciones y prácticas en los contextos de los que provienen los estudiantes.

-Aprendizajes de gestión de paz/bienestar a partir de la experiencia de la formación en la UVI.

Respecto a los postulados epistemológicos y metodológicos, la propuesta conjuga elementos derivados de las nociones teóricas que hemos desarrolladas en torno a la decolonialidad y la interculturalidad y otros, que a continuación sintetizamos. En principio, nuestra propuesta se adscribe al planteamiento realizado en la UVI respecto a la investigación vinculada en lo relativo a varios de sus criterios. Por una parte, apuesta por la construcción de conocimiento desde la convergencia de miras epistémicas diversas cuyos horizontes de sentido no son, necesariamente, convergentes; a razón de ello, no da por sentado una referencia universal y transhistórica de paz y violencia, sino que explora las nociones que en un contexto específico se tienen de ello. Por otra parte, la utilización del teatro, los títeres y los dibujos comentados obedecen, en estas coordenadas, a la necesidad de proponer un escenario abierto a la posibilidad de la construcción 
de narrativas por parte de las niñas y los niños para que expresaran, mediante historias elaboradas por ellos, los sentidos conferidos al bienestar/conflicto.

La propuesta apunta al diálogo interactoral, interlingüe e intercultural (Dietz, 2009); por ello mismo y de acuerdo con las claves analíticas que nos proporcionan los estudios decoloniales, presentadas párrafos antes, pone en duda las indagaciones que sobre la educación para la paz se han elaborado a partir de postulados construidos a priori y al margen de los contextos, los territorios, las personas y su horizonte cultural y lingüístico, y que soslayan los sentidos del bienestar/conflicto (y sus derivaciones) y las prácticas locales de gestión y negociación derivadas de ello.

La propuesta cuestiona las interpretaciones, por lo general eurocentradas, que obvian preguntarse por las nociones que actores, no necesariamente hablantes de lenguas dominantes ni formados en el canon de las disciplinas, construyen y circulan en los contextos particulares. A contrapelo, nuestra investigación pone en diálogo las nociones que estudiantes, docentes, grupos de vinculación de la UVI y la propia investigadora tienen sobre el objeto de estudio, y reconoce la relevancia de colocar en el centro del análisis los sentidos significados y los sentidos que los actores hablantes de diferentes lenguas otorgan a estas nociones.

Por último, el eje vertebrador de la propuesta de investigación es la perspectiva de género que desde los feminismos decoloniales y comunitarios (Paredes y otras, citadas en Gargallo, 2015) se ha propuesto para el análisis de la intersección de formas de dominación y violencia, que en el sistema mundo colonial y capitalista se traducen en prácticas y signos específicos de intervención y mediación patriarcal (Gutiérrez, 2014; Gutiérrez, Sosa y Reyes, 2014), cuyo andamiaje está sostenido en un conjunto de dispositivos tendentes a devaluar, desautorizar y disminuir lo femenino, feminizado y racializado.

\section{YEKYETOLISTLI Y TEIXPANOL: NOCIONES LOCALES DE BIENESTAR Y CONFLICTO}

Los talleres con los grupos de vinculación y los estudiantes de la UVI con objeto de indagar las nociones locales sobre paces y violencias, presentes en cuatro horizontes de sentido y acción, a decir: el interpersonal, el comunitario, el escolar y el familiar, nos permitieron observar lo que a continuación exponemos. En los grupos de vinculación, compuestos por niños y niñas de primarias bilingües, de edades oscilantes entre los siete y doce años, todos provenientes de los municipios de Tequila y Tlaquilpa, no se hizo alusión a los conceptos de paz y violencia para denominar las situaciones escenificadas o representadas, sino a las nociones nahuas yekyetolistli y teixpanol, alusivas a un estado de bienestar contrario al de conflicto, respectivamente. Yekyetolistli, como forma de estar en bienestar, se acompaña, desde la perspectiva de las niñas y los niños de pakilistli (felicidad) y en teixpanol está asociada a yolkokolistli (tristeza o "malestar en el corazón"), mahmawilistli (miedo) o kualanilistli (enojo).

Del mismo modo, encontramos que los horizontes de sentido y acción elegidos por los grupos de vinculación fueron el comunitario, el familiar y el interpersonal. Las historias elaboradas en las escenificaciones teatrales, con títeres y en los dibujos, tienen varios elementos en común; el primero referente al ámbito elegido por las niñas y los niños; el segundo concerniente a los actores inmiscuidos en la 
relación bienestar-conflicto-bienestar; el tercero relativo a las formas de resolución y gestión del conflicto para la reparación del daño; y el último vinculado a las razones que, desde su perspectiva, dieron origen a la afrenta.

En primer lugar, es de resaltar que en todas las historias construidas, teixpanol como forma variada de conflicto, se introduce por medio de una acción que irrumpe para desequilibrar una colectividad, ya que pone en riesgo su bienestar. Tanto en las representaciones teatrales como en los títeres, todas las narrativas tenían como hilo conductor la desestabilización de una situación colectiva, que implicaba que un personaje tomara algo que le "pertenecía" a la comunidad. Aquí es importante señalar que el sentido de pertenencia indicado no puede comprenderse como sinónimo de propiedad, sino alusivo a un bien que es de todos (en concreto, el agua, la fiesta, el cerro y las semillas) y que la comunidad requiere para preservar yekyetolistli. La afrenta representada por las niñas y los niños consistió, entonces, en el despojo o lesión de ese bien; el agua o el cerro en dos de los casos; en el sabotaje, en el caso de la fiesta; y en el robo, en el de las niñas.

El segundo elemento en común es el relativo a los personajes que inoculan conflicto y a quienes gestan su resolución. En la totalidad de las historias imaginadas por los grupos, quien afrenta, despoja y roba es un varón o un personaje masculinizado representado por un animal, y quienes gestionan la resolución de conflicto son grupos de mujeres. Solo encontramos en uno de los dibujos elaborados por niñas de Tlaquilpa una historia en la que era la abuela quien "lastimaba" mediante gritos y generaba mahmawilistli (miedo). Sin embargo, esta abuela no era propiamente asociada al conflicto en la familia, sino que despertaba temor en los niños de su núcleo doméstico.

El tercer elemento recurrente refiere a las formas propuestas por las niñas y los niños para reparar el daño y restaurar el bienestar alterado por el conflicto. En ninguno de los casos la resolución sugerida fue punitiva ni implicó castigo, sino la gestión de bienestar mediante la comprensión de las razones que llevaron a los agresores a cometer actos lesivos de su comunidad. Como analizamos en otro momento (Moreno, 2018) y como se corrobora también en otras investigaciones sobre interlegalidad y pluralismo jurídico (Sierra, 2004), estas formas de relacionamiento empáticas se sustentan en la idea de que quien lesiona, despoja o roba, generando dolor o penuria en otros, lo hace por algún motivo que, a su vez, le produce dolor.

Así, la resolución del conflicto desde esta lógica empática pasa primero por conocer las razones que llevaron a la persona a dañar a su comunidad o a sus cercanos, y segundo, por ayudarlo a resolver las causas de su agresión. Lejos de separar al agresor de su comunidad, las niñas y los niños propusieron restituir el lazo rasgado mediante una práctica socioafectiva cuya finalidad era volverlo a vincular con su comunidad.

Por último, el cuarto elemento presente en los relatos consiste en dos tipos de razones aducidas por las niñas y los niños para explicar las situaciones conflictivas. La primera de ellas, de índole afectivo, relacionada con emociones como mahmawilistli (miedo), yolkokolistli ("malestar en el corazón") o kualanilistli (enojo), que llevan a los agresores a cometer acciones como, por ejemplo, tomarse el agua de todos, sabotear la fiesta de la comunidad, quemar el cerro, todas estas acciones lesivas no eran de las personas en particular, sino de toda la gente y más allá, incluyendo otras formas de vida, como el cerro y los animales que lo habitan. 
Por otra parte, la segunda razón identificada detrás del acto disruptor era la necesidad de conseguir dinero para la resolución de una problemática. Sin embargo, en todos los casos que se exponía este argumento económico se explicaba que el dinero era requerido para responder a una necesidad de salud de un familiar cercano al agresor. Nunca se mencionó que el dinero sería para incrementar la riqueza u obtener prestigio, sino que en el horizonte de sentido de las niñas y los niños, el robo de personas (no hablaron de secuestro), o la imposibilidad de pagar una deuda, obedecía a las condiciones de precariedad de quienes hurtan y despojan, que les impide atender los problemas de salud de los suyos y por ello "deciden" hacerlo.

En respuesta a estas situaciones, todos los grupos propusieron medidas reparativas vinculadas a restituir los lazos de una comunidad por medio de la fiesta, como espacio restaurador y propicio para la gestión de conflicto; ayudar a los agresores a conseguir los recursos económicos para resolver los problemas de salud de sus familiares, de manera que pudiesen, a su vez, estar en condiciones de reparar el daño hecho; amparar emocionalmente a quien sentía envidia, tristeza o se sentía solo y que, por ello, generaba dolor y daño a otros; restaurar un vínculo lesionado entre personas mediante un "regalo, como hacen las señoras", práctica muy común entre la población nahua de la región.

Respecto a los talleres realizados con los alumnos/as de la UVI, los horizontes de sentido y acción elegidos fueron el escolar, el interpersonal y el familiar, y quedó fuera el comunitario, a diferencia de los niños y las niñas, que fue el ámbito más abordado. El curso de la reflexión giró en torno a la violencia de género, respecto a la cual identificaron las instituciones, los discursos, los dispositivos y los actores que la perpetran y reproducen.

De este primer ejercicio, resaltan la familia y la iglesia como instituciones que, desde la perspectiva de las jóvenes, son constrictivas, y cuyos mandatos de género las restringen de manera diferenciada en comparación con sus pares varones. Claramente, observan una transformación sustancial de los imperativos de género presentes en estos ámbitos respecto a lo que sus madres y sobre todo sus abuelas experimentaron en lo referente a ampliar las fronteras del ejercicio de decisiones y derechos. Sin embargo, consideran que, en la última década, dos fenómenos han mediado los procesos que organizan, significan y reproducen las prácticas productoras de género: la presencia de grupos delincuenciales en sus entornos cercanos y la creciente imposibilidad de las familias rurales e indígenas por asegurar sus condiciones de subsistencia.

Es relevante notar que, en el horizonte de sentido de los estudiantes de la UVI, la universidad representa una institución en la que es posible en paralelo afirmar aquellos aspectos que consideran son característicos de su identidad como pueblo nahua mediante un proceso de resignificación intracultural, al tiempo que cuestionar interculturalmente y desde una mirada crítica y no heteronormada prácticas, representaciones y sentidos propios de sus comunidades, que los constriñen y cercan, sobre todo en lo relativo a los dispositivos constructores de género.

Del análisis de los talleres con los alumnos/as, destacamos los siguientes resultados. En primer lugar, la UVI como proyecto pedagógico político representa, para gran parte de los alumnos/as de la sede estudiada, un espacio propicio para 
el despliegue de una reflexión crítica sobre aquellos aspectos de su propia cultura que les interesa resguardar y aquellos que es crucial revisar a la luz de un proyecto histórico propio. Al respecto de las violencias convergentes en torno a los procesos interrelacionados de racialización, generización y diferenciación de clase, este ejercicio reflexivo del propio horizonte cultural ocurre, además, en medio de la tensión del cuestionamiento de las herramientas teórico-conceptuales y epistemológicas que ofrece la universidad para "pensarse".

Esta reflexión es un campo fértil para la exploración desde la convergencia de las perspectivas intercultural, interseccional y decolonial, ya que permiten problematizar de qué manera la imbricación de formas diferenciadas de dominación se ven atravesadas, además, por factores de orden sociocultural, económico y político, lo que se traduce en expresiones y respuestas frente al poder colonial, patriarcal y capitalista igualmente diferenciadas a razón de las matrices culturales e históricas de las que los pueblos abrevan. Sin embargo, y a pesar de la prolífica producción de textos que retoman uno u otro planteamiento, no identificamos estudios que busquen la convergencia de estas perspectivas, por lo que, en potencia, este es un campo de indagación novedoso y pertinente.

De ello deriva, en segundo lugar, un cuestionamiento sagaz sobre la impronta colonial de la universidad como institución eurocentrada, en general, y sobre las posibilidades de erigir un pensamiento contrahegemónico para revisar la propia historia como pueblos indígenas en el seno de ella. Las alumnas y los alumnos reconocen en la UVI, sin embargo, un espacio abierto al pensamiento crítico, que les ha permitido elaborar una mirada propia sobre el devenir de sus pueblos, al tiempo que los ubica dentro de una historicidad mayor.

En el caso específico del análisis de las violencias, es notable el esfuerzo por trascender la substancialización de la cultura como aspecto configurante de la violencia de género: aun cuando se hace necesario explorar los códigos, las instituciones y los dispositivos de control y disciplinamiento que se gestan al interior de los grupos sociales y que se vehiculan por medio de configuraciones culturales específicas, también se requiere acudir a una perspectiva que alumbre las relaciones y los arreglos de orden económico que son detonantes de relaciones de género y, a la inversa, que permita advertir cómo estas se encuentran permeadas por relaciones económicas. En este sentido, argumentamos la necesidad de explorar cómo se imbrican las relaciones de opresión, de orden fundamentalmente político-cultural, con las de explotación, de naturaleza económica.

Ello se tradujo en un ejercicio de identificación territorializada de aspectos interrelacionados con los procesos de construcción de desigualdades, diferencias y opresiones de género, que acaecen en medio de relaciones económicas y políticas, por ejemplo. Esto fue claro al identificar cómo la constante precarización a la que están expuestas las familias campesinas e indígenas tiene derivaciones divergentes para los hombres y las mujeres que las componen, en tanto ambos están sometidos también diferencialmente en sus núcleos domésticos.

Respecto a ello, en tercer lugar es importante hacer notar que tanto niñas y niños como jóvenes llegaron a la misma conclusión por medios diferentes: en sus comunidades, la violencia que ejercen los varones hacia otros varones y hacia las mujeres de su entorno tiene como engranaje central la continua desposesión y precarización 
a la que están expuestos, que les impide responder a los imperativos a su género de aprovisionar a sus núcleos domésticos y que es un signo plenamente moderno, indicativo de cómo se trenza en el capitalismo patriarcal una suma de explotaciones, despojos y opresiones.

Este resultado no nos permite, sin embargo, aseverar que la violencia de género es unicausal y tiene como explicación unívoca la precariedad. En consonancia con lo señalado por Segato $(2006,2007,2011,2016)$, la precariedad, en sentido amplio, connota, además de la imposibilidad de acceder a un trabajo que permita asegurar bienestar y reproducir las condiciones materiales de vida, la disolución de vínculos comunitarios e interpersonales y la incapacidad, en ese sentido, de empatía, arraigo y comunalidad. En otros textos (Moreno, 2018) ya hemos abundado sobre las implicaciones de esto.

Por último, las premisas que alientan el enfoque intercultural se hacen notorias en el conjunto de argumentos que exponen los estudiantes al ser interpelados sobre los aprendizajes y las experiencias derivadas del trabajo que realizan con sus grupos de vinculación en la UVI. De esto, resaltan dos aspectos: por un lado, la constante alusión a la necesidad de construir una pedagogía de la empatía, habilitante de formas de relación vinculantes, no jerarquizantes y no escindidas con personas provenientes de horizontes culturales diferentes al propio, así como con las de su misma comunidad; y por otro, la centralidad de poner en el núcleo de la concepción de bienestar el resguardo de la vida en todas sus formas, que en medio del entre fuego del sistema de valor dinero, propio del colonial capitalismo, se encuentra en constante vulneración.

\section{CONCLUSIONES}

En este trabajo hemos analizado desde el feminismo decolonial y los estudios interculturales la contribución que hace una iniciativa de educación superior alternativa como la UVI para construir una pedagogía que detona relaciones empáticas y restaura vínculos comunitarios que los conflictos patriarcal-capitalistas han destruido o debilitado en las últimas décadas. En el caso de la región Grandes Montañas del centro de Veracruz, hemos ejemplificado cómo niñas y niños tanto como jóvenes universitarios nahuas significan desde sus comunidades y cotidianidades las paces y las violencias que caracterizan la región y cómo, a la vez, los horizontes de sentido personal, familiar, comunitario y escolar contribuyen a fomentar prácticas que gestan bienestar y, con ello, reparan conflictos.

La elección y combinación de dos actores locales diferentes, niñas y niños nahuas que conforman grupos de vinculación de la propia UVI, por un lado, y jóvenes estudiantes de esta universidad intercultural, por otro, ilustra que la educación superior intercultural puede y debe contribuir, de manera decisiva, al despliegue de nuevas relaciones horizontales, dialógicas y territorialmente arraigadas. La educación superior intercultural, tal como se practica en la sede Grandes Montañas de la UVI, contribuye -en estrecha relación con actores locales y regionales- a restaurar vínculos familiares y comunitarios que han sido tensionados, precarizados o destruidos por los conflictos patriarcal-capitalistas.

Este proceso, aún incipiente, de re-encontrar, reparar y fortalecer lazos comunitarios en estrecho acompañamiento por los jóvenes estudiantes universitarios, sin 
embargo, no es posible encauzarlo desde el aula clásica de la universidad convencional, sino en el trabajo situado, localmente contextualizado, mediador y dialógico que en la UVI se denomina como "gestión intercultural", un tipo de gestión y vinculación que docentes, estudiantes y egresadas/os de la universidad intercultural realizan en su región y en sus comunidades.

\section{REFERENCIAS BIBLIOGRÁFICAS}

Ameglio, Pietro y Ramírez, Tania (coords.) (2016). ¿Cómo construir la paz en el México actual? Textos, autores y preguntas sobre construcción, educación y cultura para la paz. México: Universidad del Claustro de Sor Juana/Plaza y Valdés Editores.

Assis, Danilo (2018). Tres momentos de la política masculinizante en el patrón de de poder colonial/capitalista. Revista Realidad, núm. 1.

Crenshaw, Kimberlé (2012). Cartografiando los márgenes: interseccionalidad, políticas identitarias, y violencia contra las mujeres de color. En Raquel Platero (Lucas) (ed.). Intersecciones: cuerpos y sexualidades en la encrucijada (pp. 87-12). España: Ediciones Bellaterra.

Dietz, Gunther (2017). Interculturalidad: una aproximación antropológica. Perfiles Educativos, vol. 39, núm. 156, pp. 192-207.

Dietz, Gunther (2009). Los actores indígenas ante la "interculturalización" de la educación superior en México: ¿empoderamiento o neoindigenismo? Revista Latinoamericana de Educación Inclusiva, vol. 3, núm. 2, pp. 55-75.

Delgado, A. (2018, 23 de agosto). Seis estados concentran el 40\% de feminicidios en el país. El Universal. Recuperado de https://www.eluniversal.com. $\mathrm{mx} /$ estados/seis-estados-acumulan-40-de-feminicidios-en-el-pais

Espinosa, Yuderkys (2014, marzo-abril). Una crítica descolonial a la epistemología feminista crítica. El Cotidiano, núm. 184. Recuperado de https://www. redalyc.org/pdf/325/32530724004.pdf

Espinosa, Yuderkys et al. (eds.) (2014). Tejiendo de otro modo: feminismo, epistemología y apuestas descoloniales en Abya Ayala. Colombia: Editorial Universidad del Cauca.

Federici, Silvia (2010). Calibán y la bruja. Mujeres, cuerpo y acumulación originaria. España: Traficantes de Sueños.

Galtung, Johan (2003). Paz por medios pacíficos. Paz y conflicto, desarrollo y civilización. Bilbao: Bakeas, Gernika Gogoratuz.

Gandhi, Mahtma (2016). Programa Constructivo, su significado y lugar. San Luis Potosí: El Colegio de San Luis.

Gargallo, Francesca (2015). Feminismos desde Abya Yala: ideas y proposiciones de las mujeres de 607 pueblos en Nuestra América. México: Universidad Autónoma de la Ciudad de México.

Grosfoguel, Ramón (2013). Racismo/sexismo epistémico, universidades occidentalizadas y los cuatro genocidios/epistemicidios del largo siglo XVI. Tabula Rasa, núm. 19, pp. 31-58.

Gundara, Jagdish (2000). Interculturalism, education, and inclusión. Londres: Paul Chapman Educational Publishing. 
Gutiérrez, Raquel (2014). Políticas en femenino. Reflexiones acerca de lo femenino moderno y del significado de sus políticas. En Margara Millán (coord.). Más allá del feminismo: caminos para andar. México: Red de Feminismos Descoloniales.

Gutiérrez, Raquel, Sosa, María Noel y Reyes, Itandehui (2014). El entre mujeres como negación de las formas de interdependencia impuestas por el patriarcado capitalista y colonial. Reflexiones en torno a la violencia y la mediación patriarcal. Revista Heterotopías, vol. 1, núm. 1. Recuperado de https://revistas.unc.edu.ar/index.php/heterotopias/article/view/20007

Hale, Charles (2002). Does multiculturalism menace? Governance, cultural rights, and the politics of identity in Guatemala. Journal of Latin American Studies, vol. 34, núm. 3, pp. 485-524.

Hooks, bell (2004). Mujeres negras. Dar forma a la teoría feminista. En Otras inapropiables. Feminismos desde las fronteras. España: Traficantes de Sueños.

Jabardo, Mercedes (ed.) (2014). Feminismos negros, una antología. Madrid: Traficantes de Sueños.

Lomnitz Adler, Claudio (1995). Las salidas del laberinto: cultura e ideología en el espacio nacional mexicano. México: Joaquín Mortiz.

Lugones, María (2012). Subjetividad esclava, colonialidad de género, marginalidad y opresiones múltiples. Pensando los feminismos en Bolivia. Bolivia: Conexión Fondo de Emancipación, serie Foros 2.

Lugones, María (2008, julio-diciembre). Colonialidad y género. Tabula Rasa, núm. 9, pp. 73-101.

Marcos, Sylvia (2014). Feminismos en camino descolonial. En M. Millán (coord.). Más allá del feminismo: caminos para andar. México: Red de Feminismos Descoloniales.Mohanty, Chandra (2008). Bajo los ojos de occidente. Academia feminista y discurso colonial. En L. Suárez y Aída Hernández (eds.). Decolonizando el feminismo: teorías y prácticas desde los Márgenes (pp. 112-161). Madrid: Cátedra. vol., núm. pp.

Moreno, Verónica (2016). Mujeres ahorradoras del centro de Veracruz y sus estrategias por la reproducción del vivir y para la disposición de sí, en medio de procesos de empobrecimiento, explotación y opresión. Tesis de doctorado en Sociología, Benemérita Universidad Autónoma de Puebla, México.

Núñez, Kathia (2017). Construcción identitaria de niños y niñas en dos comunidades indígenas desde sus discursos y prácticas en tres ámbitos de sentido: la comunidad, la casa y la escuela. Tesis de doctorado en Investigación Educativa, Universidad Veracruzana, México.

Pérez Orosco, Amaia (2014). Subversión feminista de la economía. Aportes para un debate sobre el conflicto capital-vida. Madrid: Traficante de Sueños.

Pimienta, Alejandro y Pulgarín, Raquel (2017). Educación para la paz en la escuela. Aportes de la educación geográfica y la cartografía social. En E. Sandoval (ed.). Geografías al servicio de los procesos de paz: análisis global, reflexión y aporte desde el contexto latinoamericano. Lima: Universidad Mayor de San Andrés.

Podestá Siri, Rossana (2007). La escuela y sus mundos interculturales. Hacia una propuesta metodológica de autoría infantil. En Historias, saberes indígenas y nuevas etnicidades: antología. México: Centro de Investigaciones y Estudios Superiores en Antropología Social. 
Segato, Rita Laura (2016). Patriarcado: del borde al centro. Disciplinamiento, territorialidad, y crueldad en la fase apocalíptica del capital. En La guerra contra las mujeres. Madrid: Traficantes de Sueños.

Segato, Rita Laura (2011). Género y colonialidad: en busca de claves de lectura y de un vocabulario estratégico descolonial. En Karina Bidaseca y Vanesa Vázquez Laba (comps.). Feminismos y poscolonialidad. Descolonizando el feminismo desde y en América Latina. Buenos Aires: Ed. Godot.

Sierra, María Teresa (coord.) (2004). Haciendo justicia. Interlegalidad, derecho y género en regiones indígenas. México: CIESAS/Miguel Ángel Porrúa/ Cámara de Diputados-LIX Legislatura.

UNAM (s.f.). Cultura UNAM. Recuperado de http://www.grandesmaestros. unam.mx/curso-disponible/gandhi-katha/

Universidad Veracruzana (s.f.). Observatorio Universitario de Violencias contra las Mujeres. Recuperado de https://www.uv.mx/apps/cuo/ouvmujeres/

Universidad Veracruzana-UV-Intercultural (s.f.). Criterios de investigación vinculada para la gestión. Recuperado de https://www.uv.mx/uvi/criterios-deinvestigacion-vinculada-para-la-gestion/

Von Werlhof, Claudia y Behmann, Mathias (2010). Teoría crítica del patriarcado. Hacia una ciencia y un mundo ya no capitalistas ni patriarcales. Frankfurt: Peter Lang International Verlag der Wissenschaften. 